\title{
Image Security Performance Analysis for SVM and ANN Classification Techniques
}

\author{
P.Karthika, P.Vidhya Saraswathi
}

\begin{abstract}
Image Security has been talked about the classification extemporized in numerous structures and utilizing distinctive systems just as innovations. The upgrades continue including the quickest security refreshing the system framework. This proposes a representation for verifying the video framework alongside the system and upgrades it more by relate AI methods SVM (support vector machine) and ANN (Artificial Neural Network). Both the methods are utilized together training and testing classification to produce results which are fitting for investigation reason and subsequently, turn out to be the achievement for security.
\end{abstract}

Keywords: IoT Security, Machine Learning, Artificial Intelligence, Support vector machine, Artificial neural network, Training and Testing Classification.

\section{INTRODUCTION}

The system security has been perception as 1970's and was the first concentrated as an order. Presently discussing the present circumstance the progression has taken a gigantic jump, presently unique virtual products and relevance are accessible can be utilized to continue your information, framework and organize secure. There are different security methods being utilized nowadays and these incorporate recognition frameworks, avoidance frameworks, firewalls, against infections and so on. So the questions regardless of they are adequate to distinguish and maintain the secure unblemished our system framework? The response to that question rotates roughly a novel strategies that assistance to upgrade or extemporize the current systems. The device or on the other hand identification framework can filter the framework $100 \%$ precisely, so they require an option where the extent of improvement can increment.

The methodology planned here will be an improvement where the relevance of AI can be watched. The system talked about here is utilizing the benefits of AI methods which are Support vector machine (SVM) and Artificial neural Network (ANN). The strategies are both directed learning systems and extremely have some extra highlights which can be able to make the framework progressively resistant to the assaults or dangers. AI has played a noteworthy job in

Revised Manuscript Received on December 05, 2019.

* Correspondence Author

P.Karthika*, Department of Computer Applications, Kalasalingam Academy of Research and Education, Krishnankoil, Tamil Nadu.

Email: karthika.p@klu.ac.in

P.Vidhya Saraswathi, Department of Computer Science and Information Technology, Kalasalingam Academy of Research and Education, Krishnankoil, Tamil Nadu. Email: vidhyasaraswthi.p@gmail.com maintenance of the security framework unto utilizing its knowledge component would monitor practically a wide range of dangers. The meaning of AI was given by the first its designer Arthur Samuel in 1959 as a "Field of concentrate that enables PC to learn without being unequivocally modified" [1]. Yet, the real issue here emerges how proficient can $\mathrm{AI}$ systems can end up being for verifying the host or system? The procedures planned here are SVM and ANN which are administered knowledge arrangement procedures and previous depends on straight grouping system while the later is a probabilistic support arrangement.

Video cut limitation comprises of distinguishing video fragments in a video stream. This is significant for genuine applications, for example, identifying copyright issues in video stages. Dealing with the copyright of the colossal number of recordings transferred regular is a basic test for video stages. There are a few procedures to manage video restriction, for example, a few methodologies consider bipartite diagram coordinating to gauge video cut closeness with an objective video stream; notwithstanding, these methodologies don't adapt to the significant issue known as close copy video cut/duplicate discovery.

Close copy video duplicates are those video duplicates gotten from a similar unique duplicate, by some worldwide change, for example, video re-arranging and shading moving, or some nearby changes, for example, outline altering. Different strategies manage video duplicates by utilizing standards of transient consistency, which are hard to defeat without essentially debasing the client's review experience-contrary to the privateer's objectives. These methodologies exploit another transient element to list a reference library in a way that is strong to mainstream spatial and fleeting changes in pilfered recordings. Nonetheless, they have impediments, for instance a privateer could transiently smooth a video's inclination highlights to conceal the abrupt brightening changes utilized by these strategies.

\section{PROPOSED METHODOLOGY}

To preprocess the informational collection and in request to think about on various calculations embed the preprocessed information into ANN for example Neural Network preparing and a different set into SVM classification for example Support Vector Machines preparing. Both the procedures are appropriate their strategy to follow with the training and testing dataset. 


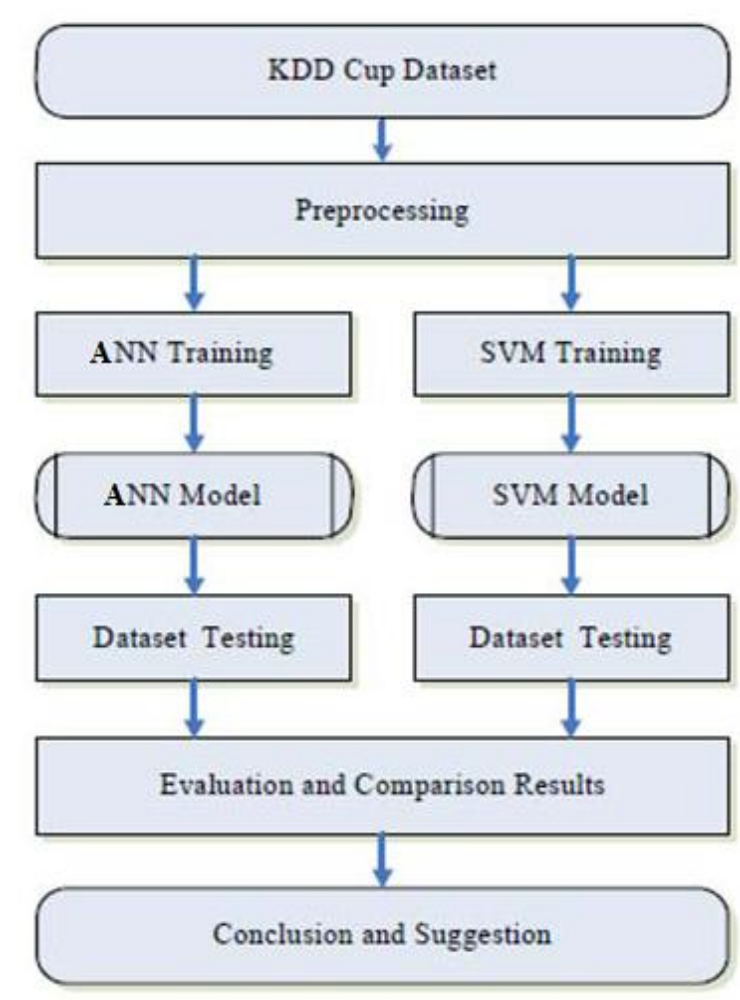

Fig. 1.Training and Testing Classification Architecture

- We locate the primary edge (stay outline) from the source video that matches in the objective video.

- Then we run a neural system based article classifier and get the items on the pictures from the source video. For our tests we utilized TFSlim + SSDLite + MobileNet_V2 $+\mathrm{COCO}$ as is advantageously lightweight and utilized for continuous applications.

- Once the articles are identified we convert them to images of a letter set.

- Then, we track the articles found in the grapple casing of the source video in the objective video, propelling edge by casing until no item is available (as enough edges have passed and the article is no longer inside the scene of the objective video).

The quantity of casings progressed on the source video to coordinate the one in which the item was lost in the objective video, this casing is utilized as the new grapple outline. By skipping edges and utilizing grapple outlines, we abstain from looking at and handling outline by edge, which would be excessively expensive as far as execution.

\section{A. Machine Learning Algorithm and its Techniques}

Good Define the AI is the rising strategies in security and connected over numerous different areas as well. It significantly classified into regulated and unverified learning strategy. Regulated learning the information to realized what sort of information it's available to order. Directed learning have a sub two class relapse and order where previous characterized can be constant assessment of shortly based on the preparing data set (perceptions and estimations) which are be relevant of new information.

The two methodologies client for order is Support Vector
Machine (SVM) and Artificial Neural Network (ANN) procedures. SVM is the knowledge machines that mark every vector class it's an exceedingly dimensional space. The essential straight engineering of SVM is known in fig 1 support vectors is notice by the highlight is chosen for the order and hyper plane is characterized that straightly the information into nosy and non meddling informational indexes separately.

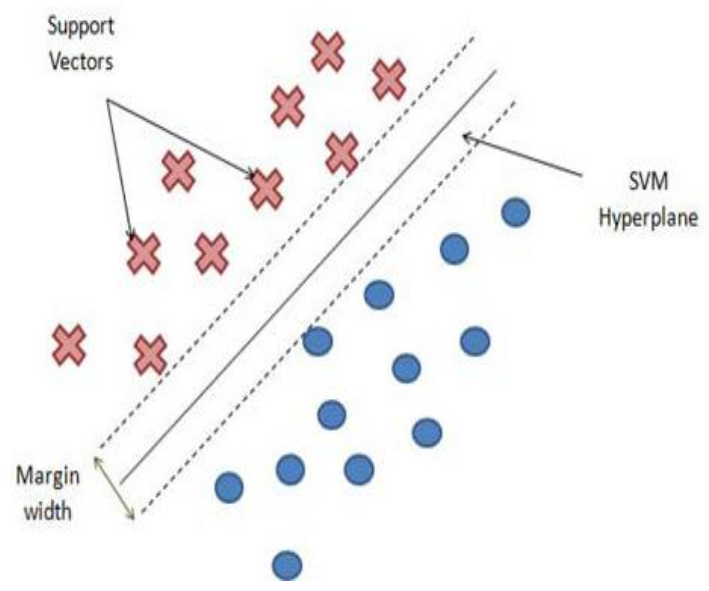

Fig. 2.SVM Classification Method

\section{B. Hypothetical Procedure of the SVM Classification}

1. Congregate the system data from the web and framework data from the separate system.

2. Process the accumulated data regarding their qualities or on the other hand header data.

3. Based on the characteristics separate the element for examination or examination.

4. Based on the highlights found in stage 3, the calculation will be connected to order the system parcel or framework data.

5. The characterization of the information in sorts of the two classes, class A relating to typical bundles/3 behaviour while the class $\mathrm{B}$ relates to unusual bundles/ 3 behaviour.

\section{SVM Classification Algorithm}

Any The Straight Classification (SVM system): It calculation plays out the capacity of separating the log records (have just as organize) Support Vector Machines are a standout amongst the most prominent machines learning calculation including input of the grouping informational collection. SVM gives the conventional system of the surface in hyper plane to the information using a bit work. The client may give a capacity of the SVM's amid to preparation of the process selects to help of the vectors outside of this work. The info SVM is given to the preparation informational collection that requirements to be prepared with the assistance help of some vectors having a place to arrangement of the info information. 


\section{$\mathrm{D}=\{(\mathrm{Xi}, \mathrm{Yi}) \mid \mathrm{Xi} \varepsilon \mathrm{RP}, \mathrm{Yi} \varepsilon\{1,-1\}\}$ (from i to $\mathrm{n}-1$ )}

Where, $\mathrm{Yi}$ is also 1 or -1 , showing the point of the class $\mathrm{Xi}$ has a place. We have to locate the greatest edge hyperplane that the points of separates it's contain $\mathrm{Yi}=1$ from those include $\mathrm{Yi}=-1$ and $\mathrm{Rp}$ is the capacity of the hyper plane is mapped. Likelihood Neural Network classifications probabilistic of advance characterizes the log documents being sifted by the SVM manner the even interruption or an example can be followed. PNN or probabilistic of the neural system design is an interesting also, stand-out in essentials from other neural system procedures.

The condition for shrouded hub work is the straightforward result of the two vectors ( $E$ is precedent vector $\mathrm{F}$ is the information include vector)

hi $=\mathrm{Ei} \mathrm{F}$

The class yield enactments are as per the following:

$$
c_{j}=\left(\sum_{i-1}^{W} e_{i}^{(h-1 / j)}\right) / N
$$

$$
\begin{aligned}
& \text { Where, } \mathrm{N}=\text { precedent vector } \\
& \text { hi = concealed hub enactment } \\
& \mathrm{Y}=\text { smoothing factor }
\end{aligned}
$$

The significant advantage of ANN that the no preparation is requires while the precedent vectors go about the concealed layer of the system.

\section{Training and Testing Classification}

The after the text edit has been completed, the paper is ready for the template. Duplicate the template file by using the Save As command, and use the naming convention prescribed by your conference for the name of your paper. In this newly created file, highlight all of the contents and import your prepared text file. You are now ready to style your paper; use the scroll down window on the left of the MS Word Formatting toolbar.

The methodologies are connected over the testing which is assembled from live condition. As saw from writing audit and by exhaustive examination to realize that SVM and ANN together can be upgraded security to improvement the space over different strategies. The theory of positive future extension of the preparation and testing to be advanced more and greatest assault data can be assembled from such exist informational index. Aside from SVM and ANN other AI systems can be viewed data set hypothesis and so forth the planned approach can be extemporized by apply cross breed innovation for instance consolidating the upsides of the distinctive systems.

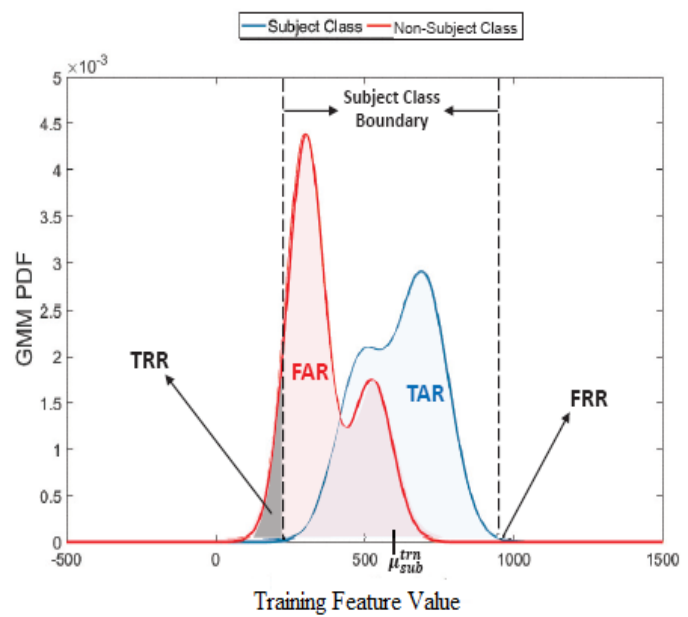

Fig. 3.Training and Testing SVM Classification

Given: $\mathrm{N}$ labeled training examples (xi, yi) with yi $2\{-1$, 1) and

xi $\in X$, and an initial distribution D1(i) over the examples.

For $\mathrm{t}=1 \ldots \mathrm{T}$ :

Train a weak classifier ht: $X \rightarrow\{-1,1\}$ using distribution Dt.

Calculate the error of $h t: \in t=P N$

$\mathrm{i}=1 \mathrm{Dt}(\mathrm{i}) 1($ yi $6=\mathrm{ht}(\mathrm{xi}))$.

Set

$\mathrm{t}=-1$

$2 \log (\in t(1-\in t))$.

Set Dt+1(i) $=\operatorname{Dt}(\mathrm{i}) \exp (\propto \operatorname{tyiht}(\mathrm{xi})) / \mathrm{Zt}$,

Where $\mathrm{Zt}=2 \sqrt{ }(\in \mathrm{t}(1-\in \mathrm{t}))$ is a normalization factor. Output the strong classifier $\mathrm{H}(\mathrm{x})=\operatorname{sign}(\mathrm{f}(\mathrm{x}))$, where

$$
\mathrm{f}(\mathrm{x})=\sum_{t=1}^{T} \alpha_{t} h_{t}(x)(x)
$$

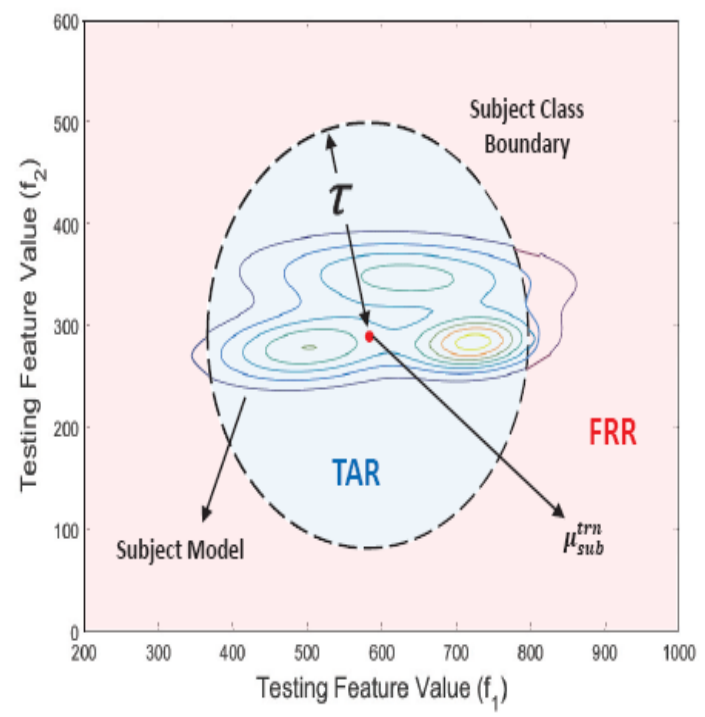

Fig. 4.Splite data Training and Testing Classification 
Measurement of each classifier and $\mathrm{m}$ is the quantity of preparing tests. Note that the mistake to test does not rely upon the number of frail classifiers in Fig 4. Therefore, general strategies for preparing dataset are to: (1) increment the quantity of preparing tests, (2) diminish training blunder and (3) decrease the intricacy of the frail classifiers. As is run of the mill [2], we process each feeble classifier from a solitary element. Here we use choice stumps (threshold highlights). Stretching out the component mining worldview to genuine esteemed powerless classifiers.

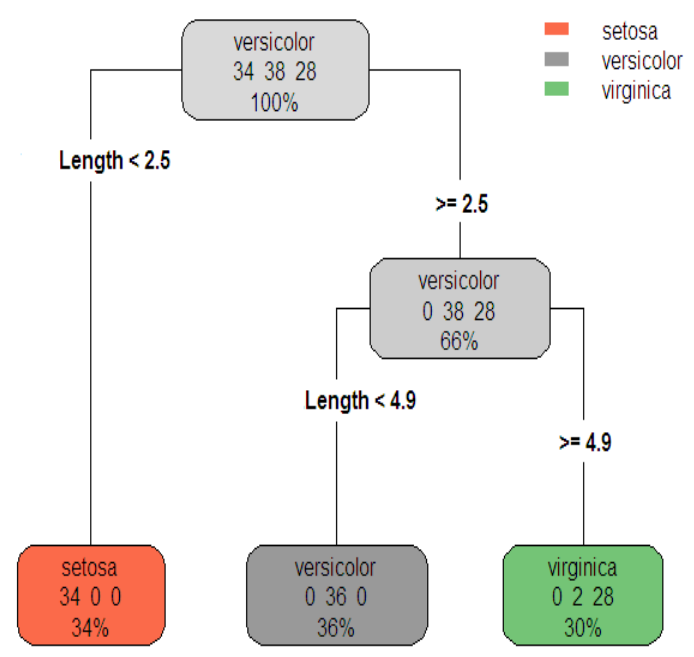

Fig. 5.Training ANN Classification

Machine Learning is to developing the procedures in security and connected over numerous different areas as well in Fig 5. It significantly sorted into directed and unverified learning procedure. Directed learning the information is recognized what sort of information it's leaving to group. Directed knowledge having the two sub classifications relapse and grouping can be characterized as nonstop assessment of the preparing on the data set (perceptions and estimations) which is related to over the new information. These two methodologies client for grouping is Support Vector Machine (SVM) and Artificial Neural System (ANN) methods.

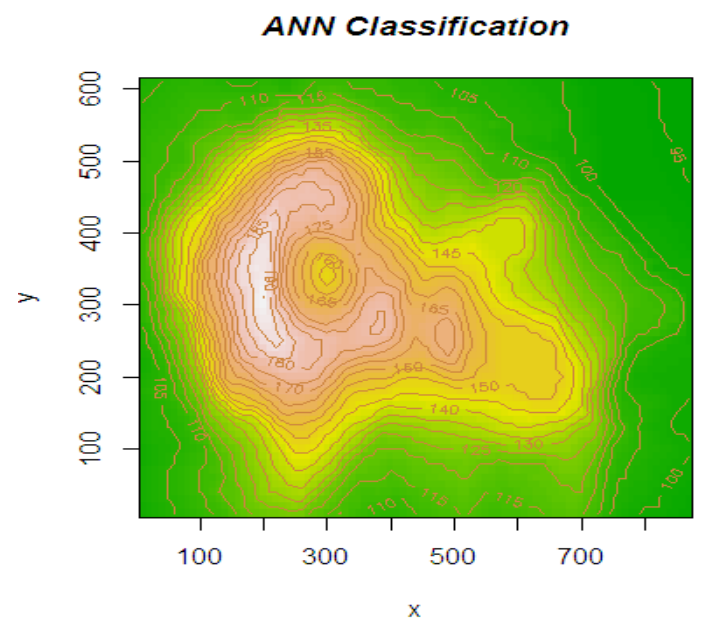

Fig. 6.Testing ANN Classification
ANN is the knowledge machines that name every vector class in a very dimensional break and over the preparing vectors plot in Fig 6. The essential direct design of ANN is specified where maintain vectors are notice by which the highlight is chosen for the characterization and a hyper plane is characterized that straightly groups the information into meddling and non meddlesome informational collections individually.

\section{IMPLEMENTATION AND RESULTS ANALYSIS}

The fundamental point of the proposed work was assessed at first by watching the genuine issues of security organism confronted and the examination work organism performed on the equivalent. Step by step the work was shaped into an increasingly explicit target where we chosen to set up an instrument similar to utility for the clients focusing on the store every single occasion being worked on the machine or in excess of the system.

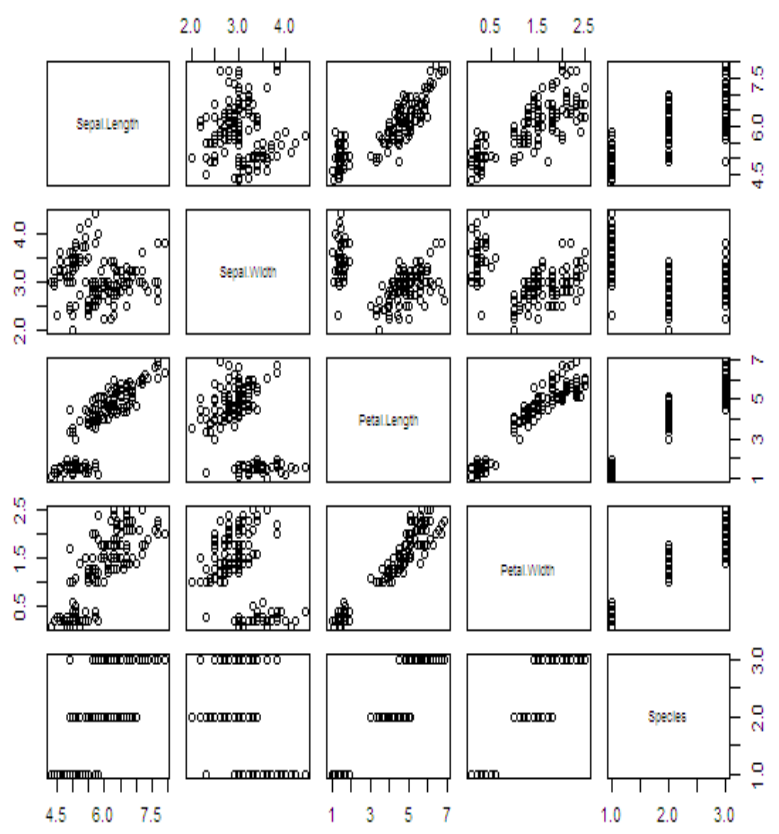

Fig. 7.Comparison Results for ANN and SVM Classification

The work begun by first considering different systems creature connected to distinguish and anticipate different security dangers over host just as organize Fig 7. To our perception, it was AI grabbed our eye, it has various strategies and approaches not exclusively can be connected on any framework. Learning section which was very amazing in AI procedures. The Among various methods are different advantages and downsides which we considered and arranged to be valid two calculations together to make the best usage of both systems in an effective way. 


\section{ANN and SVM Classification}

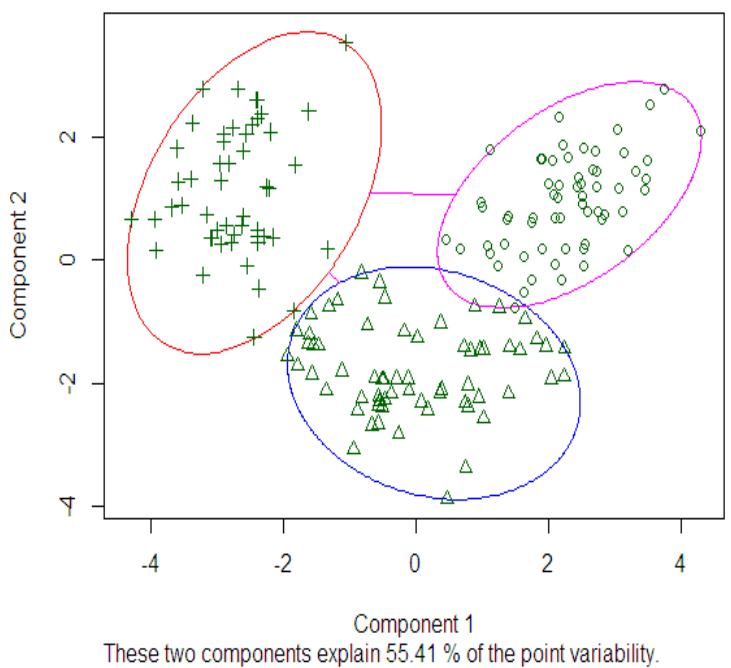

Fig. 8.ANN and SVM Classification

The regulated method first watched all the systems which go under investigated. Then exhaustive amendment of the considerable number of methodologies two was most attractive which neural system procedure and backing vector machine system in Fig 8. Both methodologies have the possess advantages and downsides yet we connected them two jointly which contributing our framework in a creative structure. Neural system approach utilized is the probabilistic a plausible esteem is picked and connected with each info neuron for instance while bolster vector machine then again is acting like straight classifier where the arrangements is done based on help vectors being picked. Both the procedures are being connected for different sorts of logs which are caught from a subsist condition.

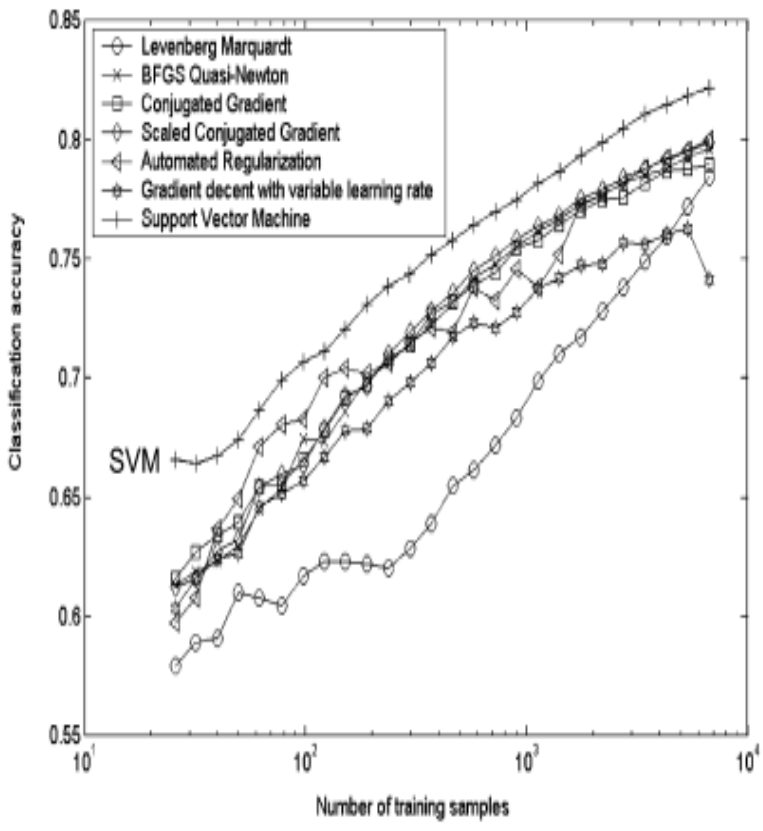

Fig. 9.Prediction accuracy of SVM classifiers optimized by average cross-validated in various training schemes
A general perception was the way that grouping exactness altogether improved with an expanding number of preparing tests, achieving a level in exhibition somewhere in the range of 2000 and 3000 examples in Fig 9. The exactness bends speak to practically perfect learning conduct. It ought to be referenced that the execution level watched does not mirror an intrinsic bunching of the informational index, as preparing information subsets were haphazardly chosen from the pool.

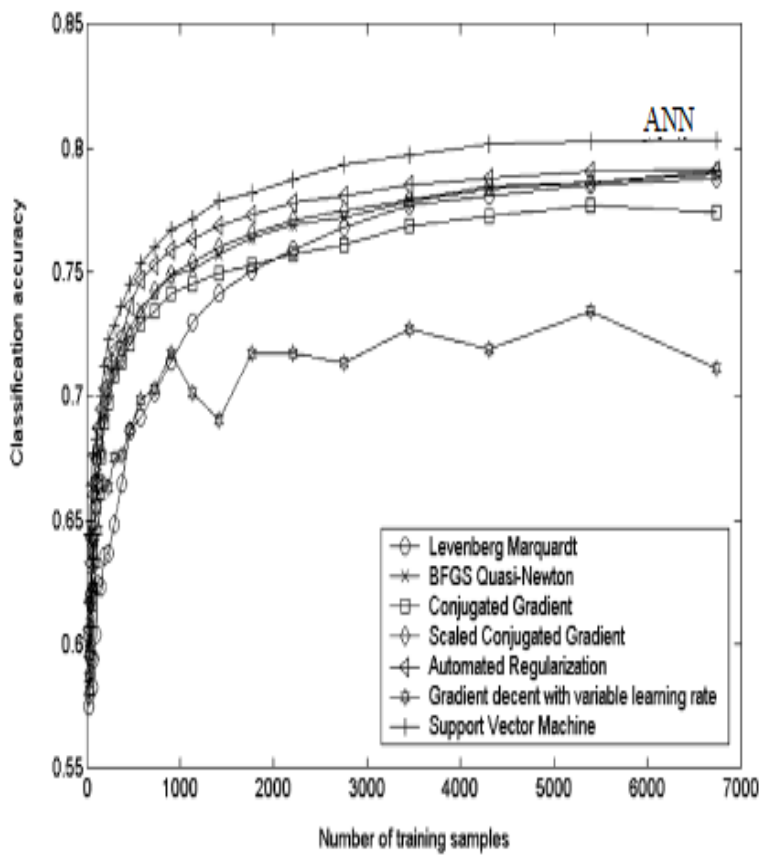

Fig. 10. Prediction accuracy of ANN classifiers optimized by average cross-validated in various training schemes

The portion effectively anticipated develops from roughly $65 \%$ to $80 \%$ when the preparation set is expanded by a factor of $\sim 250$. The descriptors improved arrangement exactness by roughly two percent one for SVM and another one for ANN contrasted with models dependent on the individual descriptors. In Fig 10 results show an ideal ANN preparing to a substantial degree relies upon the quantity of preparing designs accessible furthermore, the sort of sub-atomic descriptors utilized.

In a past correlation of machine learning SVM to a few strategies and associates it is appeared that a SVM classifier outflanked other standard and strategies, be that as it may, an exceptionally planned and basically streamlined neural organize was again better than the SVM model test. This perception is upheld of the perception that the present of investigation the arrangement of the effectively grouped by both SVM and ANN (common genuine positives) was $72 \%$ all things considered, and then division mistakenly ordered in the two frameworks (common false negatives) was $11 \%$. 
The $10 \%$ of test information were the accurately anticipated by the SVM yet flopped by ANN, and the $6 \%$ were effectively ordered by the ANN yet not by SVM utilizing the full arrangement of the descriptors.

It was exhibited that the SVM framework utilized in this think about has the ability to create higher by and large expectation precision than a specific ANN design. In view of this perception we reason that SVM speaks to a helpful strategy for order undertakings in demonstrating and virtual screening, particularly when extensive quantities of information factors are utilized. The SVM classifier appeared to supplement the expectations gotten by the ANN. The SVM and ANN classifiers gotten for medication similarity forecast are practically identical in generally speaking precision and the produce covering, yet not indistinguishable sets of accurately and misclassified mixes.

A comparable perception can be made in the two models are looked at. Diverse ANN designs and preparing calculations were appeared to prompt changed arrangement results. Along these lines, it may be insightful to apply a few prescient models are parallel, independent of the temperament, i.e., being SVM-or ANN-based. To pressure that our examination does not legitimize the end that SVM beats on ANN when all is said in done. In present work just a standard for the feed-forward system and fixed number of the shrouded neurons was contrasted with the standard of SVM execution. All things considered, our outcomes show that arrangements gotten by SVM preparing appear to be increasingly strong with a littler standard mistake contrasted with standard ANN preparing.

Independent of the result of this examine, it is the proper decision of preparing information and descriptors, and sensible scaling of info factors that decides the achievement or disappointment of AI frameworks. The two techniques are fit to survey the value of various data set descriptor for a given order task, what's more, they are techniques for decision for quick the first sifting of compound libraries. A specific favorable position of the SVM is "meager condition of the arrangement". The implies a SVM classifier for the depends on help of the vectors, then the classifier work isn't impacted by the entire informational collection, as it is the situation for some neural system frameworks. Another normal for SVM is the likelihood to effectively bargain with an exceptionally vast number of highlights because of the misuse of portion capacities, it makes an appealing procedure for the quality chip investigation or the high dimensional substance spaces. The blend for SVM with an element choice routine may give a productive instrument to extricating synthetically important data.

\section{CONCLUSION}

The outcomes creature produced are talked about the follow passages with their showing. The underlying advance in the usage was to catch the casements that are available in each framework comprising of the data about every single occasion happening on the framework. They are fundamentally of three sorts application of the framework and security. Investigation of the performed on application security and whose work as pursues: Application catch those occasions which is the application explicit and occasions are delegated mistake, cautioning what's more and data. Then again the security $\log$ s identified with the logons of clients onto the windows was effective or not.

\section{REFERENCES}

1. Esmaeili, M.M., Fatourechi, M., Ward, R.K.: “A robust and fast video copy detection system using content-based fingerprinting” . IEEE Trans. Inf. Fore. Secu. 6, 213-226 (2011)

2. Barrios, J.M., Bustos, B.: "Competitive content-based video copy detection using global descriptors". Multi. Tool. Appl. 62, (2011)

3. Ganesh Babu, R., Amudha, V.: "Resource Allocation in QoS Scheduling for IEEE 802.16 Systems. Int. J. Sci. Inno. Eng. Tech. 50-55 (2016)

4. Haitsma, J., Kalke,T.: "A highly robust audio fingerprinting system. In: International Symposium on Music Information Retrieval". pp. 107-115 (2012)

5. Jiang, M., Tian, Y., Huang, T.: "Video copy detection using a soft cascade of multimodal features". In: IEEE International Conference on Multimedia and Expo. pp. 374-379 (2012)

6. Karthika, P., Vidhya Saraswathi, P.: "A Survey of Content based Video Copy Detection using Big Data”. 3, 114-118 (2017)

7. Karthika P., Vidhya Saraswathi P.:"Content based Video Copy detection using Frame based Fusion Technique", Journal of Advanced Research in Dynamical and Control Systems Volume 9, SP-17, Pages 885-894, Publisher Technoscience Academy(2017)

8. Lei, Y., Luo, W., Wang, Y., Huang, J.: "Video sequence matching based on the invariance of color correlation". IEEE Trans. Circu. and Syst. Video Tech. 22, 1332-1343 (2012)

9. Ganesh Babu, R., Amudha, V.: "Dynamic Spectrum Access Techniques in Cognitive Radio Networks”. Int. J. Emerg. Tech. Comp. Sci.22, 508-512 (2016)

10. Liu, L., Lu, H., Xue, X.: “A segmentation and graph-based video sequence matching method for video copy detection”. IEEE Trans. Knowl. Data Eng. 25, 1706-1718 (2013).

11. Koosha Sadeghi Oskooyee, Ayan Banerjee, and Sandeep K. S Gupta. Neuro movie theatre: "A real-time internet-of-people based mobile application". In The 16th Intl. Workshop on Mobile Computing Systems and Applications, 2015.

12. Koosha Sadeghi, Ayan Banerjee, Javad Sohankar, and Sandeep K. S Gupta.Safedrive: "An autonomous driver safety application in aware cities". In 2016 IEEE Int'l Conference on Pervasive Computing and Communication Workshops.

13. Junghyo Lee, Koosha Sadeghi, Ayan Banerjee, Javad Sohankar, and Sandeep K. S Gupta. "Enabling real-time internet-of-people 4D mobile applications". In Advanced and Trusted Computing (ATC), The 14th IEEE Conference on, 2017.

14. Javad Sohankar, Koosha Sadeghi, Ayan Banerjee, and Sandeep K. S Gupta. E-BIAS: "A pervasive EEG-based identification and authentication system". Proceedings of the 10th ACM Symposium on QoS and Security for Wireless and Mobile Networks (Q2SWinet 2015), 2015.

15. Karthika, P., Vidhya Saraswathi, P.: "Digital Video Copy Detection Using Steganography Frame Based Fusion Techniques". In: International Conference on ISMAC in Computational Vision and Bio-Engineering. pp. 61-68 (2019)

16. Javad Sohankar, Koosha Sadeghi, Ayan Banerjee, and Sandeep K. S Gupta. "Systematic analysis of liveness detection methods in biometric security systems". In Advanced and Trusted Computing (ATC), The 14th IEEE Conf. on, 2017.

17. Song, J., Yang, Y., Huang, Z., Shen, H. T., Hong, R.: "Effective Multiple feature hashing for large scale near-duplicate video retrieval". IEEE Trans. Mult. 15, 1997-2008 (2013) 


\section{AUTHORS PROFILE}
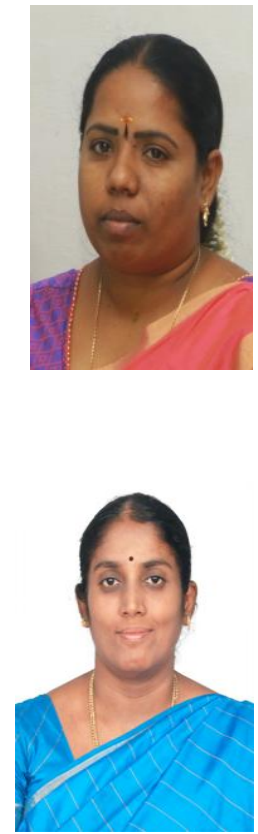

P.KARTHIKA has received BCA degree in 2008 from Trinity College for Women, Namakkal affiliate to Periyar University, Salem, MCA degree in 2011 from M.Kumarasamy College of Engineering, Karur affiliated from Anna University, Chennai. Currently she is a research fellow in the Department of Computer Applications at Kalasalingam Academy of Research and Education, Krishnankoil, TamilNadu. Research interests include Network Security with Machine Learning IoT and Steganography.

P.VIDHYA SARASWATHI has received MCA degree in 1999, M.Phil (Computer Science) degree in 2007 from Madurai Kamaraj University and Ph.D from Kalasalingam Academy of Research and Education. Her worked as a Lecturer at A,K,D, Dharmaraja Womens College, Rajapalayam, Tamilnadu, India, between 2001 and 2008.Currently, she is Assistant Professor and Head of the Department of Computer Science and Information Technology at Kalasalingam Academy of Research and Education, Krishnankoil,. 